\title{
Direct determination of the redox status of cysteine residues in proteins in vivo Satoshi Hara ${ }^{\mathrm{a}}$, Yuki Tatenaka ${ }^{\mathrm{b}}$, Yuya Ohuchi ${ }^{\mathrm{b}}$, Toru Hisaboria, c, *
}

${ }^{a}$ Chemical Resources Laboratory, Tokyo Institute of Technology, Nagatsuta 4259-R1-8, Midoriku, Yokohama 226-8503, Japan

${ }^{b}$ Dojindo Laboratories, 2025-5 Tabaru, Mashiki-machi, Kumamoto 861-2202, Japan

${ }^{\mathrm{c}}$ Core Research for Evolutional Science and Technology (CREST), Japan Science and Technology Agency (JST), Tokyo 102-0075, Japan

*Correspondence should be addressed to Toru Hisabori;

Fax: 81-45-924-5268 Tel: 81-45-924-5234

E-mail address: thisabor@res.titech.ac.jp

(C) 2014. This manuscript version is made available under the Elsevier user license http://www.elsevier.com/open-access/userlicense/1.0/ 


\begin{abstract}
The redox states of proteins in cells are key factors in many cellular processes. To determine the redox status of cysteinyl thiol groups in proteins in vivo, we developed a new maleimide reagent, a photocleavable maleimide-conjugated single stranded DNA (DNA-PCMal). The DNA moiety of DNA-PCMal is easily removed by UV-irradiation, allowing DNA-PCMal to be used in western blotting applications. Thereby the state of thiol groups in intracellular proteins can be directly evaluated. This new maleimide compound can provide information concerning redox proteins in vivo, which is important for our understanding of redox networks in the cell.
\end{abstract}

Keywords: thiol reagent, thiol redox status, photocleavable maleimide-conjugated DNA, cysteine, sulfhydryl, western blotting.

\begin{abstract}
Abbreviations
NHS, N-hydroxysuccinimide; AMS, 4-acetamido-4'-maleimidylstilbene-2,2'-disulfonic acid; PEG-Mal, methoxypolyethylene glycol-maleimide; PEO; biotin- $\mathrm{PEG}_{11}$-maleimide; ssDNA, single-stranded DNA; $\operatorname{Trx} \beta$, fusion protein of the cytosolic thioredoxin $h 1$ from Arabidopsis thaliana and the $\beta$ subunit of the $\mathrm{F}_{1}$-ATPase from Thermosynechococcus elongatus BP-1; NTR, NADPH-thioredoxin reductase from A. thaliana; roGFP, redox sensitive GFP derivative; GAPDH, glyceraldehyde-3-phosphate dehydrogenase.
\end{abstract}




\section{Introduction}

The redox status of cellular proteins is strongly affected by the conditions of the cell. The thiols of cysteine residues in proteins are subjected to redox-mediated modifications due to their high reactivity. The disulfide bond formed by two vicinal cysteines is a well-known redoxmediated modification of cysteines. Other modifications of the thiol group of cysteine, such as glutathionylation, S-nitrosylation, hyperoxidation, and palmitoylation, have also been reported $[1,2]$. These reversible, or occasionally irreversible, cysteine modifications regulate enzymatic activity or protein function. Therefore, these modifications have a great impact on various physiological phenomena in vivo.

In this study, we intended to develop a useful method to determine thiol redox status in vivo. For this purpose, we studied glyceraldehyde-3-phosphate dehydrogenase (GAPDH) as a model of thiol modification. This enzyme is a key enzyme of glycolysis with many moonlighting functions in mammalian cells [3,4]. GAPDH has three cysteines. Cys152, which is located at the active site, is known to be highly sensitive to oxidative modifications. When GAPDH is exposed to oxidative stress caused by oxidants, Cys152 undergoes oxidative modifications, such as glutathionylation, S-nitrosylation, and hyperoxidation, as well as intramolecular disulfide bond formation with Cys156 [5,6,7]. Multiple modifications sometimes occur concurrently. When HEK-293T cells were exposed to $\mathrm{H}_{2} \mathrm{O}_{2}$, sulfonation at Cys152 and disulfide bond formation between Cys152 and Cys156 were simultaneously observed. These oxidations resulted in the inactivation of the enzymatic activity and induced an interaction with p54nrb [7]. In contrast, $\mathrm{H}_{2} \mathrm{O}_{2}$ exposure induced reversible S-thiolation in the GAPDH found in yeast cells, which results in the induction of apoptosis [8]. In this case, the reduction of the S-thiolation product is mainly catalyzed by glutaredoxin, grx5 [9].

Maleimide reagents are generally used to assess the redox status of the thiols in a protein of interest. Because the maleimide group specifically reacts with the reduced form of a thiol, the number of bound maleimides varies depending on the redox situations of the thiols on the protein. This difference of the number of maleimide compounds incorporated is easily detected as a change in mobility on SDS-PAGE. When proteins labeled with maleimide compounds are separated by SDS-PAGE, the mobility of the modified protein depends on its molecular weight, which is dependent upon the number of bound maleimide compounds. So far, the maleimides 4acetamido-4'-maleimidylstilbene-2, 2'-disulfonic acid (AMS) [10] and polyethylene glycol- 
maleimide (PEG-Mal) [11] have generally been used to distinguish the redox states of proteins. However, determination of the number of reduced thiols is sometimes very difficult owing to the chemical properties of these modifiers. To overcome the difficulties of these maleimide compounds, we have developed the single-stranded DNA (ssDNA)-based maleimide compound (DNA-Mal) [12]. When a protein is labeled with DNA-Mal and analyzed on SDS-PAGE, DNAMal shifts its mobility by an identical $9.3 \mathrm{kDa}$ per molecule of DNA-Mal. This substantial and accurate mobility shift allowed us to directly determine the number of bound DNA-Mal molecules, and thus the number of reduced thiols regardless of the molecular size of the protein of interest.

However, DNA-Mal also has a disadvantage, just like PEG-Mal. These large chemical modifiers often affect the efficiency of protein transfer from the gel to the membrane during western blotting analysis. We suspect that the lower efficiencies observed are caused by the larger electrophoretic molecular weight of the labeled proteins. In addition, the transfer efficiency must be affected by the number of maleimide compounds bound to the protein. Accordingly, DNAMal and PEG-Mal are not applicable to western blotting methods or for the quantification of the proteins by staining. To overcome this disadvantage, we have introduced a photocleavable group between the ssDNA and the maleimide moiety and designated these molecules as DNA-PCMal. When a DNA-PCMal-labeled protein is separated by SDS-PAGE and subsequently irradiated with UV on the polyacrylamide gel, the DNA moiety is removed. Consequently, the labeled protein can be electrophoretically transferred to the membrane, generally with the same efficiency as non-labeled proteins. Thus, DNA-PCMal can be used as a ruler to titrate reduced thiols, even under western blotting conditions.

\section{Materials and Methods}

\subsection{Materials}

5'-Aminohexyl PC ssDNA was purchased from Tsukuba Oligo Service (Ibaraki, Japan). SM(PEG)2 and maleimide-PEG11-biotin (PEO) were purchased from Thermo Fisher Scientific (Massachusetts, USA). Methoxypolyethylene glycol maleimide (SIGMA-ALDRICH, Missouri, USA) with an average molecular weight of 5,000 Da was used as PEG-Mal in this study. Antibodies against human GAPDH and penta·His were purchased from GeneTex (Los Angeles, USA) and Qiagen (Venlo, Netherlands), 
respectively. $\mathrm{CF}_{1} \gamma$-subunit antibody was prepared in a previous study [13]. All other chemicals were of the highest grade commercially available.

\subsection{Synthesis of DNA-PCMal}

The nucleotide sequence of 5'-aminohexyl PC ssDNA was designed to be the same as that of DNA-Mal (5'-TACCTCTCCCTAACTACACAACCT-3'). The introduction of the maleimide moiety into 5'-aminohexyl PC ssDNA and the subsequent purification were performed as previously described [12], with slight modifications. After the anion exchange chromatography, the purified DNA-PCMal was desalted by ultrafiltration and then lyophilized. All procedures were performed under dark or dim light conditions. The concentrations of DNA-PCMal were determined by measuring the absorbance at $260 \mathrm{~nm}\left(\varepsilon_{260 \mathrm{~nm}}=244.3 \mathrm{mM}^{-1} \mathrm{~cm}^{-1}\right)$.

\subsection{Proteins}

The expression and purification of $\operatorname{Trx} \beta$, NTR [12], and roGFP [14] were performed as described. A gene encoding Arabidopsis thaliana methionine synthase (MetE) (AT5G17920) with his-tag appended to its N-terminus was cloned into the expression vector pET23a. E. coli strain BL21 (DE3) harboring the resulting plasmid was cultured at $37^{\circ} \mathrm{C}$ in $2 \times$ YT medium supplemented with $50 \mu \mathrm{g} / \mathrm{mL}$ ampicillin and $1 \mathrm{mM}$ $\mathrm{ZnCl}_{2}$ until $\mathrm{A}_{600}$ reached 0.7. Expression was induced by the addition of IPTG to a final concentration of $1 \mathrm{mM}$, and then incubated at $21^{\circ} \mathrm{C}$ for $16 \mathrm{~h}$. MetE protein was purified using a nickel-nitrilotriacetic acid Superflow (Qiagen) followed by a Superdex 200 (GE Healthcare, UK).

\subsection{Two-dimensional gel electrophoresis}

DNA-PCMal-labeled protein was separated using 8\% BisTris/MOPS-PAGE gel, and the gel was then soaked in $360 \mathrm{mM}$ BisTris- $\mathrm{HCl}$ ( $\mathrm{pH}$ 6.8) for $10 \mathrm{~min}$ to exchange the buffer. The lanes of the gel containing proteins were excised, placed on top of $12 \%$ BisTris gel and then fixed. A second electrophoresis was performed using MES running buffer.

\subsection{SDS-PAGE and western blotting analysis}

Proteins were subjected to neutral $\mathrm{pH}$ gel systems (BisTris/MES or 
BisTris/MOPS) to analyze their molecular weights. After SDS-PAGE, the proteins were transferred to a PVDF membrane at $100 \mathrm{~mA}$ constant current for $60 \mathrm{~min}$ using a semi-dry blotter and Towbin buffer. Following procedures were performed according to standard methods, and chemi-luminescence signals on the membrane were detected using ECL prime (GE Healthcare) and a LAS-3000mini (Fuji Film, Tokyo).

\subsection{Preparation of HeLa cells proteins and labeling}

HeLa cells at $60 \%-70 \%$ confluence were incubated in the presence or absence of redox reagents, and the cells in the dishes were then washed three times with PBS. Cold $10 \%$ TCA was directly added to fix the redox state in the HeLa cells. At this point, cell lysate was extracted from the cell and the denatured proteins were precipitated by centrifugation. The proteins obtained were immediately dissolved in the extraction buffer [50 mM Tris- $\mathrm{HCl}$ (pH 6.8), $1 \mathrm{mM}$ EDTA, $0.5 \mathrm{M} \mathrm{NaCl}, 2 \%$ SDS, and the protease inhibitor cocktail Complete (Roche, Manheim, Germany)] containing $2.5 \mathrm{mM}$ maleimide compounds, and the free thiols were labeled for $90 \mathrm{~min}$ at $37^{\circ} \mathrm{C}$. Following UV-irradiation of the electrophoresed gel for $15 \mathrm{~min}$, proteins were electrophoretically transferred to the PVDF membrane as described above. The samples were kept in the dark during the labeling and SDS-PAGE procedures to avoid undesired cleavage of DNA-PCMal.

\section{Results and Discussion}

\subsection{Constraint of DNA-Mal as a thiol-labeling modifier}

Compared with unlabeled and AMS-labeled GAPDH, we only observed a substantially weak signal from DNA- and PEG-Mal-labeled GAPDH in HeLa cells (Fig. 1A) when proteins were visualized by western blot analysis. Similar result was obtained from the spinach $\mathrm{CF}_{1} \gamma$ subunit (Fig. 1B). These weak band intensities are attributable to the low efficiency of the protein transfer process because the protein bands visualized in the PEG-Mal and DNA-Mal lanes were much weaker than those in other lanes when the total proteins on the membrane were directly stained with CBB. These results clearly indicate that these large maleimide compounds prevent the transfer from gel to membrane, rather than preventing the antigen-antibody interaction. If this is the case, removal of the large molecule used for protein labeling from the protein after SDS- 
PAGE separation must be operative. For this purpose, we employed the photocleavage reaction to detach the DNA moiety from the labeled protein [15].

\subsection{Synthesis of DNA-PCMal}

We designed a new molecule containing a photocleavable site between the DNA and maleimide moieties named DNA-PCMal. DNA-PCMal was synthesized by coupling the primary amine in 5 '-aminohexyl PC ssDNA to the NHS ester in $\mathrm{SM}(\mathrm{PEG})_{2}$. After the purification and desalting processes, DNA-PCMal showed a good yield of $>80 \%$. To confirm the molecular mass of DNA-PCMal, we performed MALDI-TOF mass spectrometry using ultrafleXtreme (Bruker Daltonics, Billerica, USA) in the positive ion mode, and obtained an observed mass of 7217.567 Da. This value is identical to the theoretical mass of the cleaved DNA, 7217.67 Da, indicating that the nitrogen-laser at $337 \mathrm{~nm}$ used for the measurement completely cleaves the PC group of DNA-PCMal. When the molecular mass of DNA-PCMal was determined using ESITOF MS using micrOTOF II (Bruker Daltonics) in the negative ion mode, a mass of 7817.04 Da was obtained, which is identical to the theoretical mass of 7817.27 Da. In addition, the cleaved DNA-PCMal (theoretical mass, $7215.67 \mathrm{Da}$ ) was detected as a fragment of $7215.59 \mathrm{Da}$ in the ESI-TOF MS. A fragment with the same molecular mass was also observed in the synthesized 5'aminohexyl PC ssDNA. Because these contaminating cleaved DNA fragments without the maleimide moiety were assumed not to prevent the thiol-maleimide reaction, we used this DNAPCMal for our following study.

\subsection{In-gel photocleavage}

To examine the in-gel cleavage, using UV-irradiation, of the DNA moiety from DNAPCMal after labeling protein thiols, we applied DNA-PCMal to model proteins containing known numbers of thiols and separated them by using two-dimensional SDS-PAGE. The first dimension was performed after labeling with DNA-PCMal, and the second dimension was performed after the removal of the DNA moieties (Fig. 2B). As a control experiment, DNA-PCMal-labeled proteins were examined without UV-irradiation (Fig. 2A). Consequently, protein spots appeared in a diagonal line on the 2D-gel. In contrast, when UV-irradiation was applied to the primary SDS-PAGE gel, spots were observed out of the diagonal line, and they lined up in a horizontal line on the 2D-gel. These exceptive spots are explained as a result of the removal of DNA moiety, 
which gives the same migration distance to the non-labeled and DNA-PCMal-labeled proteins in the second SDS-PAGE process.

This technique is applicable to the proteomic analysis of the redox states of proteins. According to a report by Muthuramalingam et al. (see Fig. 2 in [16]), we can label only the oxidized thiols in the proteome with DNA-PCMal using the method explained briefly below. Reduced thiols in the lysate are blocked with iodoacetamide or N-ethylmaleimide. Then, DTT is added to reduce the oxidized and non-blocked cysteine residues. The newly exposed thiols, which were originally oxidized, are then labeled with DNA-PCMal. When the whole proteins of interest are labeled with DNA-PCMal and then separated using two-dimensional SDS-PAGE, in a manner similar to that shown in Fig. 2, the resultant spots appear on different diagonal lines, depending on the number of thiols involved in each oxidative modification.

\subsection{Western blotting analysis}

We then examined the improvement in transfer efficiency of proteins labeled with relatively high-molecular-weight maleimide compounds using recombinant N-terminally histagged methionine synthase (MetE; Fig. 3A). Unlabeled protein was easily detected using an antiHis antibody after the transfer of the proteins onto a PVDF membrane, irrespective of UVirradiation. In contrast, no visible amounts of protein were transferred during western blotting when the proteins were labeled with DNA-Mal or PEG-Mal in advance. When DNA-PCMal was used as a thiol modifier and the gel was then irradiated with UV-light, almost the original amounts of proteins were recovered and visualized using an anti-His antibody.

DNA-PCMal was then used to detect GAPDH in the cell lysates using the western blotting technique (Fig. 3B). GAPDH in the cell lysate was labeled with thiol modifiers in advance and then separated by SDS-PAGE. Because GAPDH has three cysteines, GAPDH labeled with DNA-Mal or DNA-PCMal was detected as a distinct large band (the estimated MW, 64.9 kDa) using the western blotting method. As expected, only the band intensity of the protein labeled with DNA-PCMal was remarkably enhanced by UV-irradiation (Fig. 3B). In the HeLa cell lysate, many proteins must have free thiols that can be labeled with thiol modifiers. Therefore, we confirmed the change in transfer efficiency itself by staining the transferred membrane directly with CBB. The protein band intensities were enhanced when the proteins were labeled with DNAPCMal in advance and then irradiated with UV-light (Fig.3C). In spite of the difference in transfer 
efficiency, the same migration pattern was observed for DNA-Mal- and DNA-PCMal-labeled proteins, indicating that DNA-PCMal must add the same molecular weight to the labeled proteins as DNA-Mal does.

\subsection{Redox responses of GAPDH in HeLa cells}

To generate oxidative stress, HeLa cells were incubated for $10 \mathrm{~min}$ after the addition of $1 \mathrm{mM}$ diamide, and then fixed with TCA. SDS sample buffer containing AMS, PEO, or DNAPCMal were added to the precipitates, and the solubilized proteins labeled with these reagents were analyzed (Fig. 4A). Although we could observe the slight mobility shift of the AMS-labeled GAPDH, the extent of the shift was too small to distinguish the different conditions of the three thiols on the molecule. To separate the oxidized and reduced forms of GAPDH on SDS-PAGE and to determine the proportion of redox states, we examined PEO (M.W. 922.1 Da) and DNAPCMal. Labeling with PEO and DNA-PCMal could certainly distinguish reduced forms of GAPDH from oxidized forms (Fig. 4A). Based on the band intensities, similar proportions of oxidized GAPDH (around 20\%) were observed using both modification methods, indicating that these thiol modifiers are applicable for determining the proportion of redox states of the desired protein in the cell. However, the big advantage of DNA-PCMal is that the observed mobility shifts calculated from the reduced $(29.6 \mathrm{kDa})$ and oxidized forms of GAPDH $(8.9 \mathrm{kDa})$ can be used to determine the number of free thiols; three and one, respectively This implies that diamide treatment of HeLa cells induces the formation of a disulfide bond in $20 \%$ of the GAPDH molecules.

We then investigated the differences in the oxidation of GAPDH in vivo by diamide and $\mathrm{H}_{2} \mathrm{O}_{2}$, and following reduction by DTT, or recovery of thiols by endogenous reduction systems, such as thioredoxin reductase and thioredoxin (Fig. 4B). Whereas diamide oxidation caused disulfide formation in a portion of GAPDH in the cells, the binding of one, two, and three DNAPCMal molecules to GAPDH was observed when cells were oxidized with $\mathrm{H}_{2} \mathrm{O}_{2}$ for 20 min (Fig. 4A). These three oxidation states of the enzyme should correspond to the formation of a disulfide bond (one-DNA-PCMal binding) as suggested by the diamide experiment, one modified cysteine (two-DNA-PCMals binding), and the completely reduced state (three-DNA-PCMals binding), respectively. These results are in harmony with the report that $\mathrm{H}_{2} \mathrm{O}_{2}$-induced oxidations are initiated by transient sulfenic acid formation on a thiol and that the following oxidation results in 
sulfinic and sulfonic acid formation, or disulfide bond formation, depending on the nature of the protein and the existence/lack of vicinal cysteine residues [17]. This sulfenic acid and the disulfide bond can be reduced by thioredoxin or glutathione, and by chemical reductants, such as DTT or 2-mercaptoethanol, whereas the sulfinic acid is reduced only by sulfiredoxin in an ATP dependent manner [18]. Sulfonic acid formation is thought to be irreversible. To reveal the redox states of thiols on GAPDH when oxidized in vivo, $\mathrm{H}_{2} \mathrm{O}_{2}$ - and diamide-treated $\mathrm{HeLa}$ cells were further incubated in the presence or absence of DTT. The $44.2 \mathrm{kDa}$ band (one-DNA-PCMal binding) observed in both $\mathrm{H}_{2} \mathrm{O}_{2}$ - and diamide-treated HeLa cells easily disappeared upon incubation with DTT and removal of oxidants. In contrast, the addition of DTT after the oxidation causes almost no changes in the intensity of the $57.8 \mathrm{kDa}$ band (two-DNA-PCMal binding). These results indicate that the oxidation state of GAPDH induced by $\mathrm{H}_{2} \mathrm{O}_{2}$ is irreversible due to the formation of sulfinic or sulfonic acids (Fig. 4B).

As shown in Fig. 4A, only DNA-PCMal can separate the distinct bands that depend on the oxidative states of the thiols in GAPDH and can, therefore, quantify the proportions of redox states. The oxidation of GAPDH by diamide treatment was still going on after 20 minutes, implying that oxidation with diamide is slower than that with $\mathrm{H}_{2} \mathrm{O}_{2}$.

\subsection{Usefulness of DNA-PCMal to study the redox states of protein in vivo and in vitro}

Hence, we could distinguish the different oxidation states of the GAPDH molecule in HeLa cells treated with $\mathrm{H}_{2} \mathrm{O}_{2}$ and diamide. We could also observe the reversibility of the oxidation following reduction and recovery. The proportions of each oxidation state could be quantitated using band intensities rather than activity measurements (Fig. 4B). When HeLa cells were oxidized with diamide, a disulfide bond was formed in the molecule. Given the fact that the ratio of GSH to GSSG is significantly shifted to oxidative conditions by the addition of diamide $[19,20]$, GAPDH might be oxidized by GSSG when diamide is supplied. This must be the reason why the oxidation of GAPDH in the cell by the addition of diamide was apparently slower than the oxidation induced by $\mathrm{H}_{2} \mathrm{O}_{2}$ (Fig. 4B). In contrast, $\mathrm{H}_{2} \mathrm{O}_{2}$-oxidized GAPDH showed an irreversible oxidation state at one thiol. $\mathrm{H}_{2} \mathrm{O}_{2}$ must react directly with the thiols on the GAPDH molecule and form sulfinic or sulfonic acid, as previously reported [7]. Although we have to examine sulfinic and sulfonic acids on the protein using specific antibodies or mass spectrometry analysis of peptides, we could easily and quantitatively distinguish the oxidation states in GAPDH just using 
DNA-PCMal, as shown in Fig. 4B.

\section{Acknowledgements}

This work was supported by the Nano-Macro Materials, Devices and System Research Allianace (to S.H. and T.H.) and supported, in part, by the Core Research of Evolutional Science and Technology (CREST) program of the Japan Science and Technology Agency (JST) (to T. H.). We thank Kazunori Sugiura and Masato Koizumi for their technical supports. 


\section{References}

[1] C.E. Cooper, R.P. Patel, P.S. Brookes, V.M. Darley-Usmar, Nanotransducers in cellular redox signaling: modification of thiols by reactive oxygen and nitrogen species, Trends Biochem Sci 27 (2002) 489-492.

[2] C. Jacob, G.I. Giles, N.M. Giles, H. Sies, Sulfur and selenium: the role of oxidation state in protein structure and function, Angew Chem Int Ed Engl 42 (2003) 47424758.

[3] M.A. Sirover, On the functional diversity of glyceraldehyde-3-phosphate dehydrogenase: biochemical mechanisms and regulatory control, Biochim Biophys Acta 1810 (2011) 741-751.

[4] C. Tristan, N. Shahani, T.W. Sedlak, A. Sawa, The diverse functions of GAPDH: views from different subcellular compartments, Cell Signal 23 (2011) 317-323.

[5] I. Schuppe-Koistinen, P. Moldeus, T. Bergman, I.A. Cotgreave, S-thiolation of human endothelial cell glyceraldehyde-3-phosphate dehydrogenase after hydrogen peroxide treatment, Eur J Biochem 221 (1994) 1033-1037.

[6] L. Molina y Vedia, B. McDonald, B. Reep, B. Brune, M. Di Silvio, T.R. Billiar, E.G. Lapetina, Nitric oxide-induced S-nitrosylation of glyceraldehyde-3-phosphate dehydrogenase inhibits enzymatic activity and increases endogenous ADPribosylation, J Biol Chem 267 (1992) 24929-24932.

[7] N.R. Hwang, S.H. Yim, Y.M. Kim, J. Jeong, E.J. Song, Y. Lee, J.H. Lee, S. Choi, K.J. Lee, Oxidative modifications of glyceraldehyde-3-phosphate dehydrogenase play a key role in its multiple cellular functions, Biochem J 423 (2009) 253-264.

[8] M.R. Hara, N. Agrawal, S.F. Kim, M.B. Cascio, M. Fujimuro, Y. Ozeki, M. Takahashi, J.H. Cheah, S.K. Tankou, L.D. Hester, C.D. Ferris, S.D. Hayward, S.H. Snyder, A. Sawa, S-nitrosylated GAPDH initiates apoptotic cell death by nuclear translocation following Siah1 binding, Nat Cell Biol 7 (2005) 665-674.

[9] D. Shenton, G. Perrone, K.A. Quinn, I.W. Dawes, C.M. Grant, Regulation of protein S-thiolation by glutaredoxin 5 in the yeast Saccharomyces cerevisiae, J Biol Chem 277 (2002) 16853-16859.

[10] T. Kobayashi, S. Kishigami, M. Sone, H. Inokuchi, T. Mogi, K. Ito, Respiratory chain is required to maintain oxidized states of the DsbA-DsbB disulfide bond formation system in aerobically growing Escherichia coli cells, Proc Natl Acad Sci U S A 94 (1997) 11857-11862.

[11] H. Mori, N. Shimokawa, Y. Satoh, K. Ito, Mutational analysis of transmembrane regions 3 and 4 of SecY, a central component of protein translocase, J Bacteriol 186 (2004) 3960-3969. 
[12] S. Hara, T. Nojima, K. Seio, M. Yoshida, T. Hisabori, DNA-maleimide: an improved maleimide compound for electrophoresis-based titration of reactive thiols in a specific protein, Biochim Biophys Acta 1830 (2013) 3077-3081.

[13] K. Yoshida, Y. Matsuoka, S. Hara, H. Konno, T. Hisabori, Distinct redox behaviors of chloroplast thiol enzymes and their relationships with photosynthetic electron transport in Arabidopsis thaliana, Plant Cell Physiol 55 (2014) 1415-1425.

[14] S. Hara, T. Hisabori, Kinetic analysis of the interactions between plant thioredoxin and target proteins, Front Plant Sci 4 (2013) 508.

[15] J. Olejnik, E. Krzymanska-Olejnik, K.J. Rothschild, Photocleavable aminotag phosphoramidites for 5'-termini DNA/RNA labeling, Nucleic Acids Res 26 (1998) 3572-3576.

[16] M. Muthuramalingam, A. Matros, R. Scheibe, H.P. Mock, K.J. Dietz, The hydrogen peroxide-sensitive proteome of the chloroplast in vitro and in vivo, Front Plant Sci 4 (2013) 54.

[17] M. Lo Conte, K.S. Carroll, The redox biochemistry of protein sulfenylation and sulfinylation, J Biol Chem 288 (2013) 26480-26488.

[18] B. Biteau, J. Labarre, M.B. Toledano, ATP-dependent reduction of cysteinesulphinic acid by S. cerevisiae sulphiredoxin, Nature 425 (2003) 980-984.

[19] S.F. Martin, H. Sawai, J.M. Villalba, Y.A. Hannun, Redox regulation of neutral sphingomyelinase-1 activity in HEK293 cells through a GSH-dependent mechanism, Arch Biochem Biophys 459 (2007) 295-300.

[20] E.W. Iyamu, The redox state of the glutathione/glutathione disulfide couple mediates intracellular arginase activation in HCT-116 colon cancer cells, Dig Dis Sci 55 (2010) 2520-2528. 


\section{Figure legends}

Fig. 1. Defect in transfer efficiency caused by DNA- and PEG-Mal. (A) HeLa cell lysates were labeled with a $2.5 \mathrm{mM}$ concentration of the indicated compounds. Then $0.4 \mu \mathrm{g}$ of protein was separated by a $10 \%$ BisTris/MES-PAGE and detected using an anti-GAPDH antibody. After western blotting detection, the membrane was stained with CBB. (B) Spinach leaves were ground in liquid nitrogen and the proteins were extracted and labeled with $2 \mathrm{mM}$ of the indicated maleimide-containing extraction buffer by boiling for $5 \mathrm{~min}$, and then incubation for $60 \mathrm{~min}$ at room temperature. After centrifugation and collection of the supernatant, $10 \mu \mathrm{g}$ of the spinach lysate was separated by a $10 \%$ BisTris/MES-PAGE, and detected using an anti-CF $\mathrm{CF}_{1}-\gamma$ subunit antibody. After western blotting detection, the membrane was stained with CBB.

Fig. 2. Two-dimensional gel electrophoresis. DNA-PCMal-labeled proteins were separated by $8 \%$ BisTris/MOPS-PAGE as the first dimension and by $12 \%$ BisTris/MES-PAGE as the second dimension (A). Before electrophoresis in the second dimension, the gel was exposed to UV light for $15 \mathrm{~min}$ (B). DNA-PCMal-labeled or unlabeled proteins (DNA-PCMal-labeled and unlabeled $\operatorname{Trx} \beta$, labeled and unlabeled NTR and unlabeled roGFP) were used as markers on the $12 \%$ BisTris/MES-PAGE.

Fig. 3. DNA-PCMal recovers the signal depending on UV-irradiation. (A) Recombinant MetE was labeled using $150 \mu \mathrm{M}$ concentrations of the indicated maleimide compounds, separated by $8 \%$ BisTris/MOPS-PAGE, and then detected using an anti-penta-His-antibody (left lanes). The gel was exposed to UV light for $15 \mathrm{~min}$ before the western blotting analysis (right lanes). (B) HeLa cells lysate was labeled using $2.5 \mathrm{mM}$ concentrations of the indicated maleimide compounds, separated by 10\% BisTris/MES-PAGE, and detected using an anti-GAPDH antibody (left lanes). The gel was exposed to UV light for $15 \mathrm{~min}$ before the western blotting analysis, (right lanes). Samples containing $0.4 \mu \mathrm{g}$ of protein were applied to each lane of the gel. (C) Samples were prepared using the same methods as used in Fig. 2B. Samples (2.0 $\mu$ g of protein) were analyzed by SDS-PAGE and then transferred to a membrane. This membrane was then stained with CBB. 
Fig. 4. Visualization of redox states of GAPDH in HeLa cells. (A) HeLa cells were incubated for 10 min in DMEM medium containing $1 \mathrm{mM}$ diamide. Then, a cell lysate was collected using $10 \%$ TCA. After maleimide labeling, 10\% BisTris/MES-PAGE was used for the separation of the proteins in the lysate. The gel was irradiated with UV-light for $15 \mathrm{~min}$. (B) HeLa cells were incubated at $37^{\circ} \mathrm{C}$ for $10 \mathrm{~min}$ in DMEM medium containing $1 \mathrm{mM}$ concentrations of the indicated oxidants. The medium was then exchanged with medium containing a $1 \mathrm{mM}$ concentration of the same oxidant, with PBS, or with $2 \mathrm{mM}$ DTT, respectively, and incubated for further $10 \mathrm{~min}$. After those cells were collected in 10\% TCA, cell lysates were labeled using DNA-PCMal and detected by western blotting analysis followed by UV-irradiation. A table below the figure indicates the respective proportions of the band intensities (\%), determined using ImageJ. Asterisks denote partially degraded GAPDH. Expected conditions of the thiols on the GAPDH molecule are shown on the right side. 
A

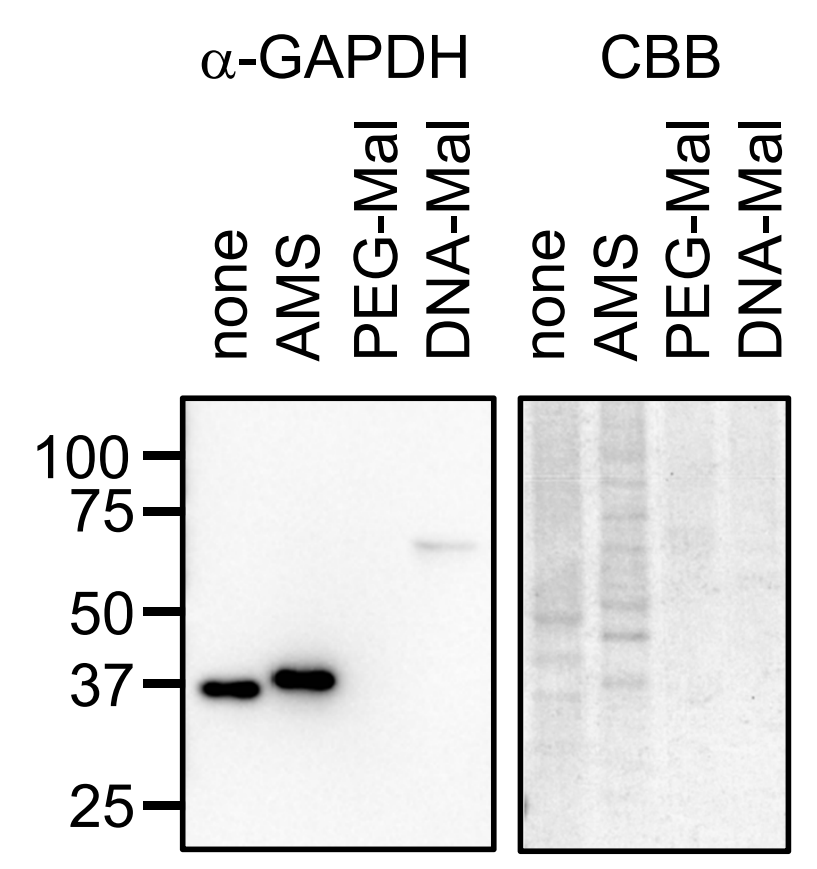

B

$\alpha-\mathrm{CF}_{1} \gamma$-subunit CBB
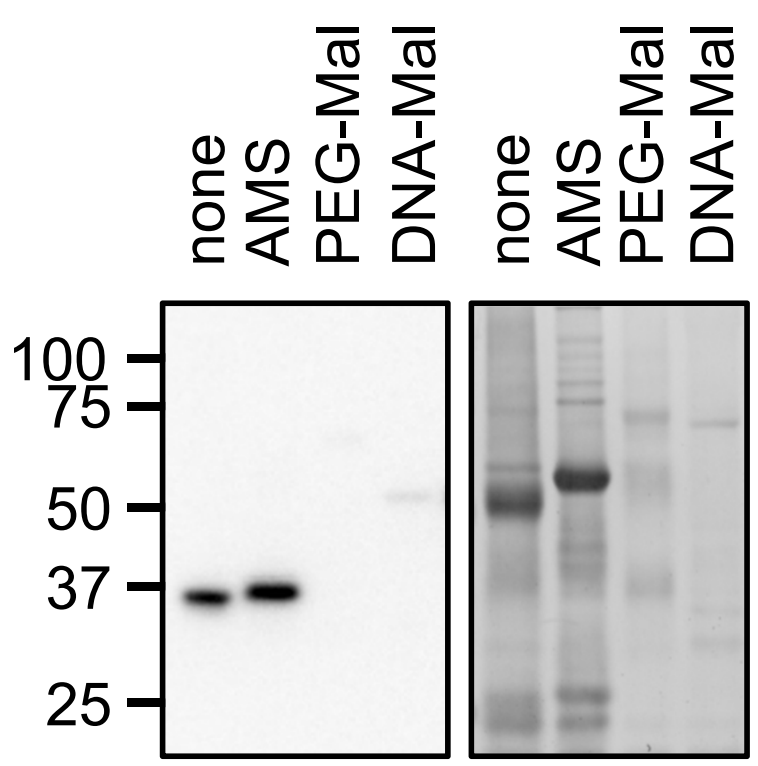

Figure 1 


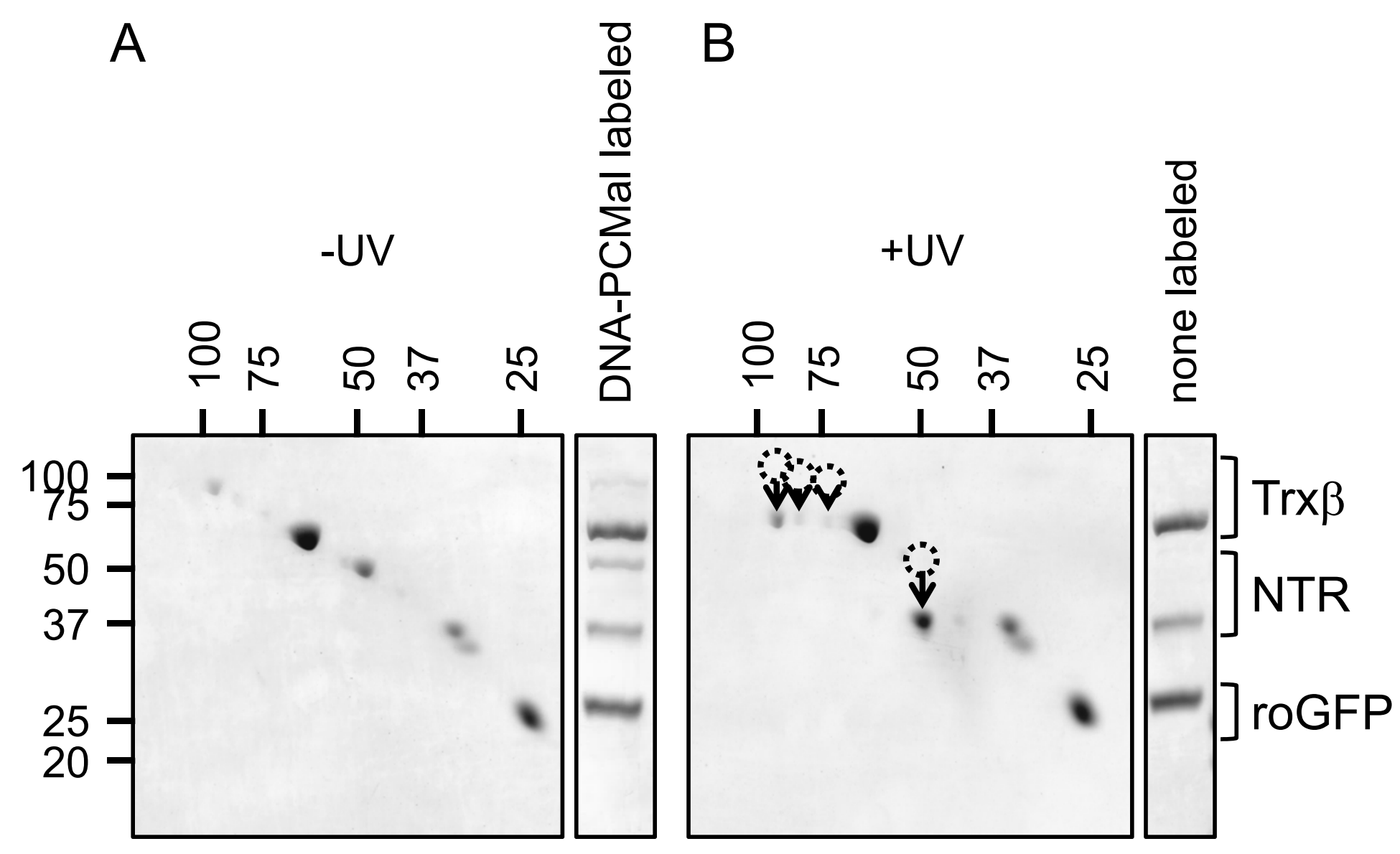

Figure 2 
A

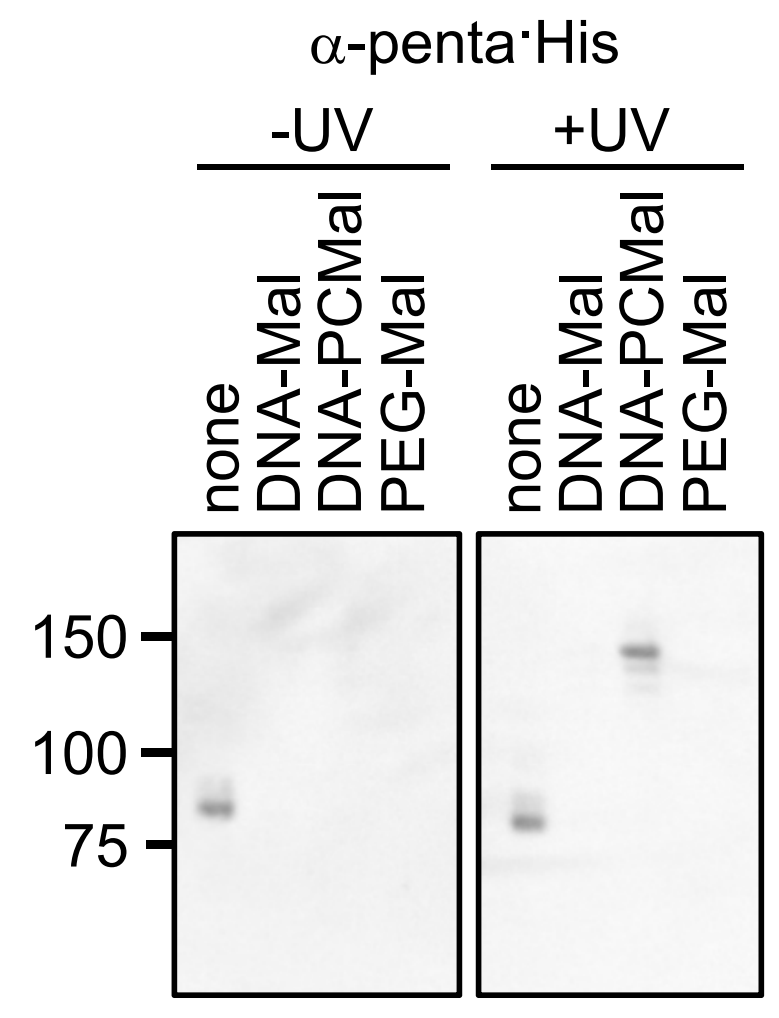

B

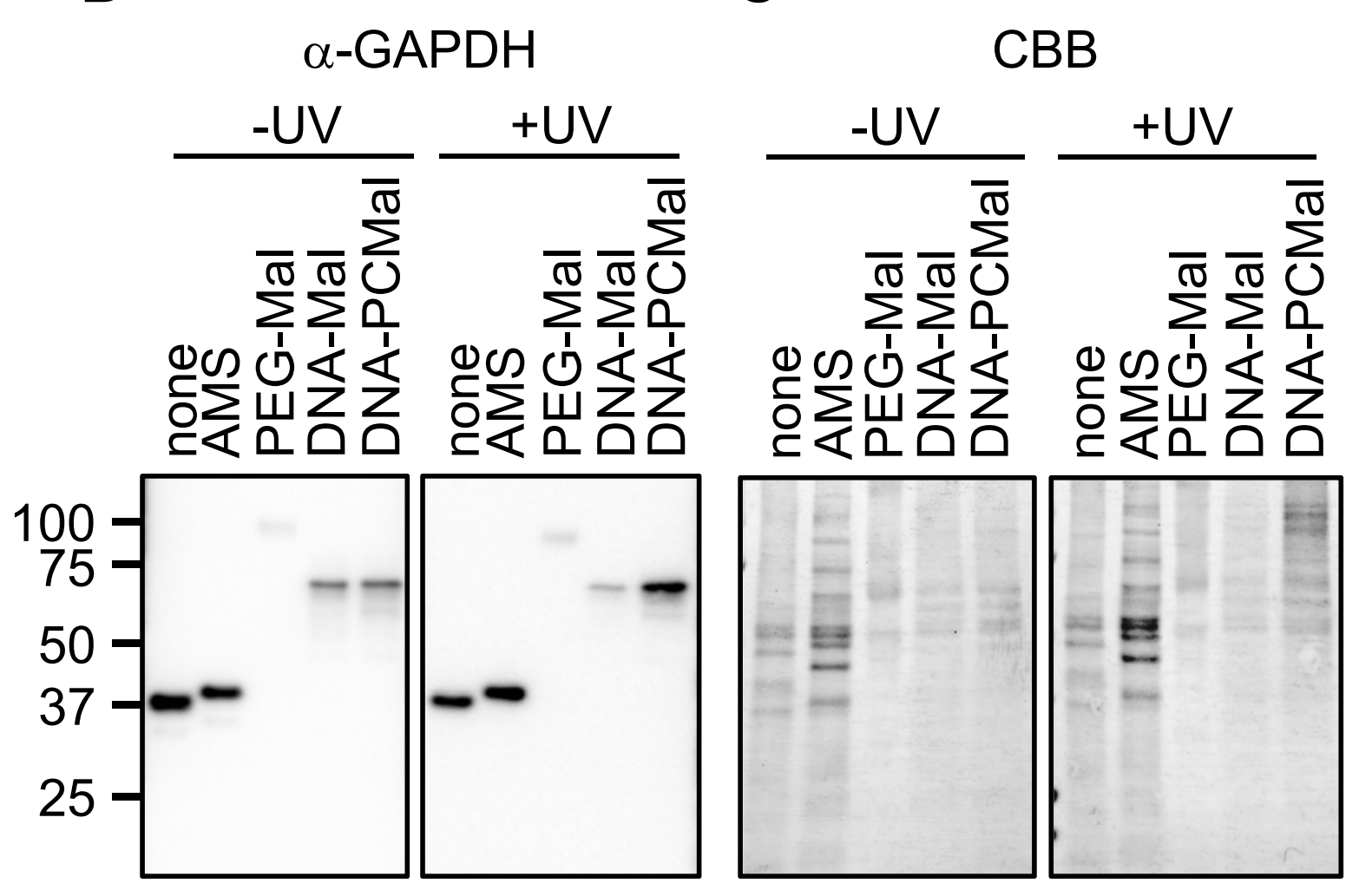

Figure 3 


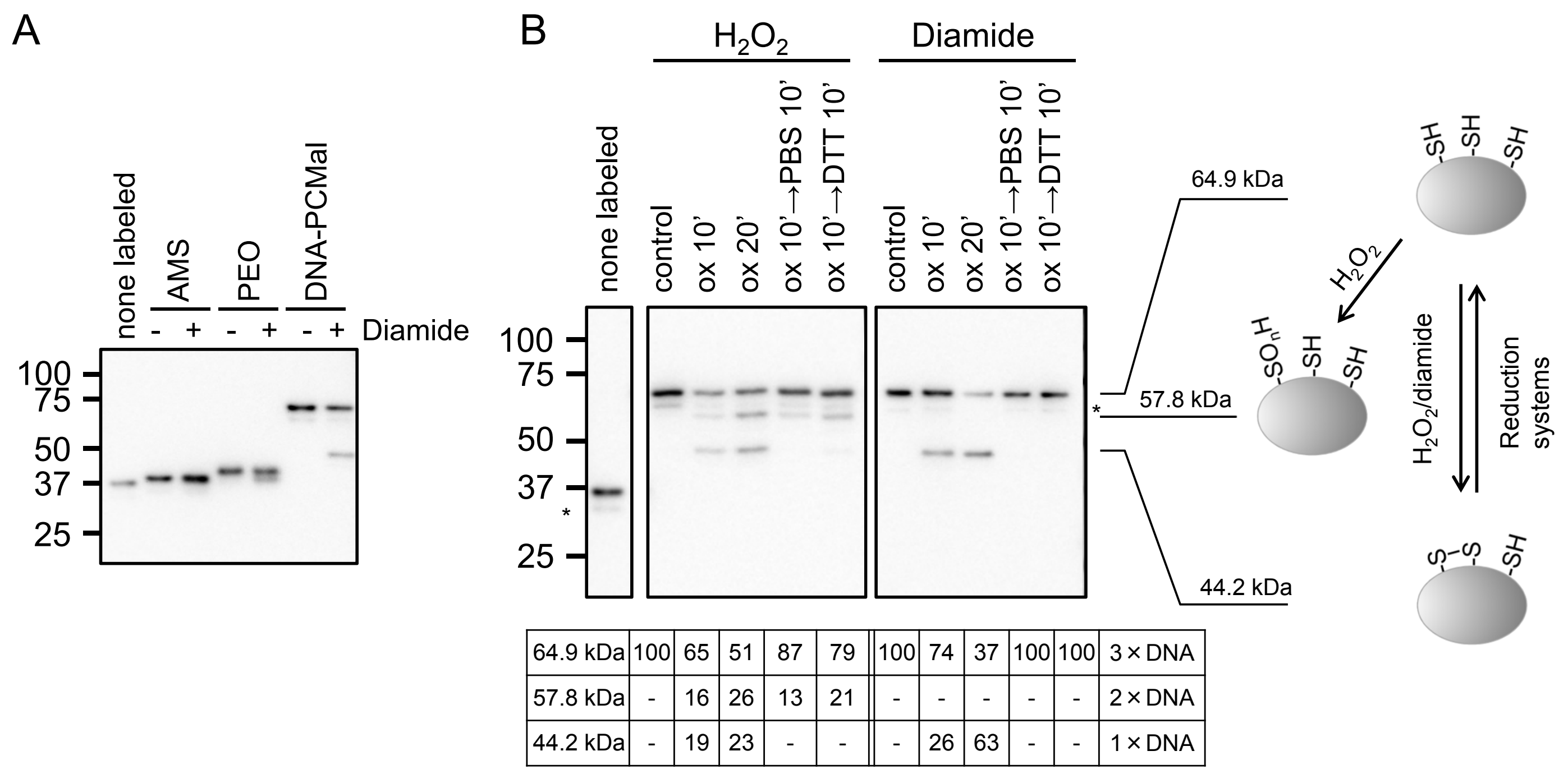

Figure 4 